\title{
Design of the Automatic Test System for Satellite Navigation Receiver Xingqi Sun ${ }^{1, a}$ \\ ${ }^{1}$ High-tech Institute,Fan Gong-ting South Street on the 12th, QingZhou 262500 , China.
}

\author{
Keywords: Navigation receiver; Parallel test; Signal imitation
}

\begin{abstract}
In order to improve the accuracy of the test of the satellite navigation receiver, the method of automatic test was adopted in this paper, and combined with PXI hardware platform, an automatic test system for receivers has been developed. This system has the function of parallel automatic test, and support the test of speed accuracy, the test of initial warm start time positioning, the test positioning accuracy, the test of speed measurement sensitivity and other tests. The speed accuracy and speed measurement sensitivity can be tested by the hardware structure and software function modules that are designed in this paper. The test result shows that acquisition sensitivity of the system is high and the speed measurement sensitivity accords with the design requirements.
\end{abstract}

\section{Introduction}

In recent years, compass satellite navigation system develops fast and performance of receivers has become a main focus. There are two testing methods. The first one is the manual test method. The other is the automatic test method. There are many uncertain factors in the former method. It can not guarantee the accuracy and its cost is very high. The latter one has characteristics of controlling efficacy, developing conveniently and low cost[1]. At present, many researchers adopt automatic test method to build the system and they have gained good achievements. With the appearance of the navigation signal simulator, the new path of research was created for automatic test of receivers.

\section{Overall Design of Automatic Test System}

The system consists of six parts, namely, test receivers, server, PXI chassis, signal input and output equipment, power divider and power supply.

By using navigation signal simulator,this system can not only repeatedly perform signal navigation, but also can not be limited by the time display and space display. It can control navigation signal reasonably according to self-requirements. The power supply is the programmable power supply, which can provide electricity for receiver performance test. Within the period of running of the power divider, this system supports serial server to run simultaneously with it. Under this circumstance, the parallel test of many receivers can be carried out[2]. The receivers have been running in a bad environment for a long time. In order to reduce the influence of environmental factors, the PXI hardware development platform of NI has been adopted in this system. The embedded controller has been applied in this system to satisfy the design requirement.

\section{Hardware Design}

In order to accelerate test speed of receivers, the programmable interface technology is introduced and also other technologies which include compatible technology and parallel test technology are introduced in this research.

There are eight slots in the inner structure of the PXI chassis. The PXI chassis can support embedded control. Based on this, the total throughput of the data will change after adding Express chassis. The throughput of data can turn into $8 \mathrm{~GB} / \mathrm{S}$ from $4 \mathrm{~GB} / \mathrm{S}$. After adding this chassis, a lot of data with complicated structure can be recorded[3]. Furthermore, it can also accelerate data transmission speed and becomes the main factors that can influence stable running of the system. As for hardware signal generation, the system can accumulate function of three kinds of boards to one board. There are three functions, which include signal closing function, radio frequency 
function and medium frequency modulation function on the board. The signal closing function can control output of signal power and signal synthesis. The radio frequency function can control frequency conversion of the signals. Medium frequency modulation function can modulate BD2 signal and GPS signal. The power supply in this test research is DP832 direct-current power supply. It can support programming, cooperate with system software for testing and control test link effectively. In addition, the DP832 direct-current power supply can provide electricity to all kinds of receivers. The $30 \mathrm{~V}$ voltage and $5 \mathrm{~V}$ voltage can also be provided. The running electric current range is from 0 to $3 \mathrm{~A}$. The corresponding maximum power is $195 \mathrm{~W}$. It is obvious that the power supply can satisfy the testing requirement of the system.

In order to improve the efficiency of data transmission, the wireless transmission method will be adopted in this system. The method can build a NIPXI testing platform by using Rf switch, DAC technology, DSP platform, embedded system, FPGA technology and frequency conversion channel. The testing platform consists of three subsystems, they are radio frequency subsystem, medium frequency modulation subsystem and embedded software subsystem. It can timely control the running system by using automatic testing software. The TCP/IP protocol can achieve the transmission of configuring scenario parameters and send the parameters to navigation receivers[4]. Then, testers will open signal simulator equipment. Testers should set navigation message and signal parameters before testing. The parameters can be transmitted to the medium frequency modulation board by PXI bus. Furthermore, in order to accelerate data transmission speed, the system adopts standard PXI bus as a bridge connecting two interfaces. One of the interfaces needs to connect with medium frequency modulation subsystem. The other one needs to connect with embedded software subsystem. Medium frequency modulation board not only modulates baseband signal carrier but also modulates the subcarrier signal or spread spectrum signal. If so, medium frequency modulation board can control level and carrier phase so that a medium frequency simulation signal can be generated. Orthogonal analog signal is the communicative bridge between radio frequency subsystem and medium frequency modulation subsystem. It has the function of transmitting data and controlling signal power and the signal frequency. Function implementation interface is $\mathrm{I}^{2} \mathrm{C}$. In order to gain a standard navigation signal, the automatic test software will be adopted in this system. The software can analyze accumulated signal data. The signal data are the main basis of evaluation of receivers' performance. The final results can be displayed.

\section{Software Design}

There are two parts in the software part of the system. One is the automatic testing software. The other is the navigation signal simulation software. The latter part can achieve timely transmission of signals based on TCP/IP communication protocol. The former part is used to configure navigation parameters and control running of instruments. In addition, it will also take charge evaluation of testing indicators and reasonable control. Within the period of system test, the most basic operating content is the configuration parameters that include programmable control power parameters, serial port parameters, test procedures and other parameters. The main content of the test includes controlling test procedures, evaluating test indicators and dealing with corresponding data. The core indicator of the software test is the conventional indicator. It can be used to test the sensitivity, positioning precision, speed precision and positioning time of the obtained data.

The designing idea of the software part of the whole system is modular design. Based on the hardware signal simulation, a complete satellite navigation signal simulator[5] can be formed by adding software signal simulation. Among these, the software signal simulation can generate observation data and observation telegraph text. These data will be taken as data resources for simulating hardware signals. In addition, the evaluation of system software also needs these data so that the authenticity of evaluation can be seen. The simulation of hardware signals mainly refers to the simulation of radio frequency signal. The equipment environment will be regarded as the environment of testing interface machines. The parameters transmitted by automatically controlled software in this system will be collected, and then they will be transmitted to navigation simulators uniformly. The parameters of every module will be configured separately and the calculation of 
satellite state and vehicle state will be completed. The calculating results can be regarded as the main basis to judge whether it is a visible star. Power parameter and navigation parameter will be transmitted to corresponding hardware driving modules after binding process. According to the navigation signal and message that are finally generated by software, its parameters have higher accuracy.

\section{Experimental Results and Analysis}

Acquisition sensitivity and speed measurement sensitivity can be tested and analyzed in this research.

Acquisition Sensitivity of the System. The testing environment of acquisition sensitivity in this system is consistent with the basic requirements. The user receiver runs at the speed of 10 meters per second and the $45^{\circ}$ direction of north by east. It belongs to low dynamic user models. The corresponding attenuation power amplitude is $1 \mathrm{~dB}$. When the position error is not beyond 50 meters, it is estimated that the indicator evaluation is effective. The testing result of acquisition sensitivity of the system is shown in the Table 1.

Table 1 Testing Result of Acquisition Sensitivity of the System

\begin{tabular}{ccc}
\hline Evaluation Results & Receiver $1(\mathrm{dBm})$ & Receiver 2 $(\mathrm{dBm})$ \\
\hline Maximum of Evaluation & -126 & -127 \\
Minimum of Evaluation & -128 & -128 \\
Variance & 0.56 & 0.21 \\
Mean value & -126.80 & -127.70 \\
\hline
\end{tabular}

Table 1 shows that the testing results of acquisition sensitivity of the two receivers are both not beyond $-128 \mathrm{dBm}$. The sensitivity ranges are basically the same. The two averages are nearly the same. The variance of the receiver 1 is greater than that of the receiver 2 . It shows that the receiver 2 can run more stably, but the two testing values can both satisfy the designing requirement of the system.

Speed Measurement Sensitivity of the System. Vehicle speed of the signal simulator will be controlled under 500 meters per second. When the speed becomes 300 meters per second, the frequency varies a lot. As a result, the speed of this test is $300 \mathrm{~m} / \mathrm{s}$. The test will be run at the $45^{\circ}$ direction of north by east. The result of horizontal speed sensitivity can be gained by changing speed. The specific results are shown in Table 2.

Table 2. Testing result of Horizontal Speed Accuracy

\begin{tabular}{ccc}
\hline Speed $(\mathrm{m} / \mathrm{s})$ & Receiver 1 $(\mathrm{m} / \mathrm{s})$ & Receiver 2 $(\mathrm{m} / \mathrm{s})$ \\
\hline 300 & 1.822 & 1.884 \\
200 & 1.031 & 1.576 \\
100 & 0.583 & 0.826 \\
50 & 0.356 & 0.332 \\
30 & 0.317 & 0.331 \\
10 & 0.463 & 0.271 \\
\hline
\end{tabular}

The data in Table 2 can be drawn into a curve in Figure 1. 


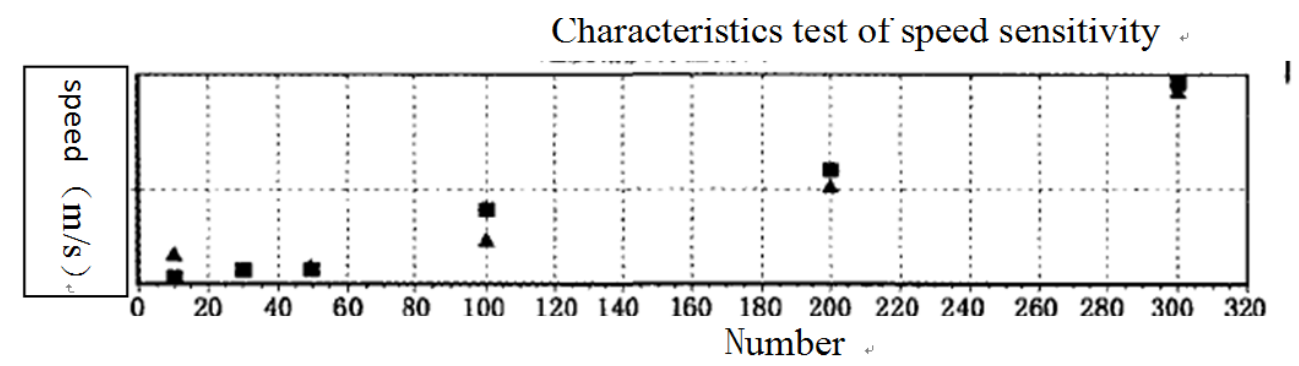

Receiver 1

$\Delta$ Receiver 2 .

Figure 1. The Accuracy Test Curve of Receiver Speed

According to Figure 1, within the fixed vehicle speed, vehicle speed measuring accuracy decreases with the increasing of speed so that the error is big. Compared with receiver 2, the error of receiver 1 is under 1.822. The performance of the receiver 1 is much better.

\section{Conclusion}

The research of the automatic test of satellite navigation receivers has been explored in this paper. An automatic test system of the receiver has been designed by adopting testing receivers, server, PXI chassis, signal input and output equipment, power distributors and power supply. The system can run stably and support parallel test. It has a better controlling function of testing procedures. According to the testing results, the acquisition sensitivity of the system is high and the speed measurement sensitivity is consistent with designing requirements. At present, the system also needs to be improved. Testing efficiency of the system will be the main research focus.

\section{References}

[1] S.L.Feng and S.Li: Design and Implementation of Automatic Test System for Multiband Reconfigurable Radar Receiver[J],Modern Electronics Technique, 2016, 39(11):13-15.

[2] D.M.Wei,F.Wang,H.C.Liu,etc:Design of a Satellite Navigation Receiver Based on LEON3 Open Source Soft Core[J],Telecommunication Engineering, 2016, 56(6):653-658.

[3] G.Huang, Y.F.Ji and X.Y.Sun: The Design of Data Acquisition System Based on the Navigation Satellite Signal[J],Electronic Technology Application,2016, 42(4):63-66.

[4] X.Xue, H.L.Qin and H.Lu: Design and Implementation of Automation Test System for GNSS Receiver[J], Journal of Navigation and Positioning, 2015, 3(4):95-99.

[5] X.L.Cao and C.J.Guo: Design and Implementation of GNSS Receiver Test System Based on Software Defined Radio[J],GNSS World of China, 2017, 42(1):49-52. 